\title{
Precision Navigation and Timing Enabled by Microtechnology: Are We There Yet?
}

\author{
Andrei M. Shkel \\ Micro Technology Office (MTO) \\ Defense Advanced Research Projects Agency (DARPA) \\ 3701 N. Fairfax Dr., Arlington, VA, USA
}

\begin{abstract}
This paper reviews currently recognized needs for advances in precision navigation and timing technology, summarizes ongoing efforts, and discusses future technological developments being pursued under the aggregated DARPA/MTO Microtechnology for Positioning, Navigation, and Timing (micro-PNT) program.
\end{abstract}

Keywords: MEMS,Inertial Sensors, Clocks, Microtechnology, PNT, DARPA, MTO

\section{INTRODUCTION}

Are we there yet? After about two decades of harmonic investment in developments, this is a question impatiently raised over-and-over by potential users of "small technology" for Positioning, Navigation, and Timing (PNT) applications. It is clear that some significant advances have been made over the years, and we see a footprint of the technology in an ever-growing consumer electronic market full of interactive products enabled by inertial and timing micro-technologies. These products include accelerometers for gaming applications, gyros for auto safety, and resonators for clocks - just to name a few. The question remains, however: Is the technology really on the level of what we consider to be precision navigation and timing, i.e., is it capable of achieving an accuracy level of at least $10 \mathrm{~m}$ in position and $1 \mathrm{~ns}$ in time throughout the entire duration of missions that may range from minutes to hours to days?

In reality, "small technology" remains several orders of magnitude short with respect to long-term stability, dynamic range, and accuracy when compared to "conventional technology," which is already known to perform adequately for many military applications. Why does making inertial instruments and clocks small necessarily lead to degradation in performance? We don't yet have a complete answer to this question, and we are still working hard to disprove the contention that "high-performance inertial micro-instrument is a contradiction in terms." It is indisputable that we can make things small, but we cannot yet make them sufficiently precise and uniform; the accuracy of lithography-based manufacturing is on the order of $10^{-2}-10^{-3}$ (the ratio of the average defect to the smallest feature size), while the accuracy of conventional manufacturing utilizing precision machining is two to three orders of magnitude higher, on the order of $10^{-5}$. We know we can deposit materials layer-by-layer with high precision, but we cannot make micro-devices truly 3 - $\mathrm{D}$, as is readily achievable using conventional machining. We consistently have an excellent case for low-cost and bulk fabrication, but we cannot seriously challenge "boutique" processes when it comes to achieving precision, structural complexity, and longterm stability. We need new knowledge regarding the dimensional stability of materials. We also need a better understanding of material scaling, surface effects, energy loss mechanisms, and the consequences of fabrication imperfections on the performance of micro-instruments.

PNT applications demand both unusual new fabrication technologies and new materials with special properties. To achieve the required phenomenal accuracy for precision navigation and timing, we need a new wave of innovation in design and refinement of many existing transducers. Future breakthroughs in microtechnology for PNT will likely rely on yet-to-be-exploited physics, new materials, highly specialized fabrication technologies and batch assembly techniques, selective wafer-level trimming and polishing, a combination of passive and active calibration techniques strategically implemented right on-chip, and introduction of innovative test technologies.

Further author information: E-mail: andrei.shkel@darpa.mil, Telephone: +1571 2184422

Micro- and Nanotechnology Sensors, Systems, and Applications III, edited by Thomas George, M. Saif Islam, Achyut K. Dutta, Proc. of SPIE Vol. 8031, 803118

(C) 2011 SPIE · CCC code: 0277-786X/11/\$18 · doi: 10.1117/12.885805

Proc. of SPIE Vol. 8031 803118-1 
This paper discusses the focused interest of the Microsystems Technology Office (MTO) of the Defense Advanced Research Projects Agency (DARPA) in the development of a miniature, self-sufficient navigation system that might be realized through deep integration of timing, inertial navigation units, and other non-inertial sensors. A new wave of innovation is underway focused on bringing to life revolutionary ideas and fabrication technologies on micro/nano/pico/femto/atto scales, packaging, ultra-low-power electronics, innovative algorithms, never-before-explored architectures, and exploitation of new integration paradigms.

\section{NEED FOR ADVANCED CAPABILITIES}

PNT technology usage has doubled every five years since 1960, mostly due to the U.S. Global Positioning System (GPS) program and the miniaturization of electromechanical components. Future PNT usage is expected to double every two years because of telecommunication, automobile navigation, robotics, and other commercial markets inserting MEMS technologies. ${ }^{1}$

The modern PNT paradigm is based on the assumption that the space-based GPS is accessible most-ofthe-time to provide position, velocity, and timing information, thereby enabling every user to operate on the same reference system and timing standard. Today's military systems increasingly rely on GPS, creating a potential vulnerability for U.S. and allied warfighters should the GPS be degraded or denied. When the GPS is inaccessible, critical information with respect to position, orientation, and timing can only be gathered through self-contained on-board instruments: a local clock and two triads of inertial sensors (three accelerometers for position and three gyroscopes for orientation). The ideal solution would be a self-sufficient instrument not relying on any external information. Precision microscale clocks and inertial sensors are required to address the paradigm of self-contained PNT. The desirable characteristics of micro PNT components are outlined in Ref. 1 and summarized in this section.

\subsection{Clocks}

Time-distribution systems provide synchronous measurements of time within a network of users. Position and time have a relationship important to a broad spectrum of military applications, including communication systems that feature efficient spectrum utilization, resistance to jamming, high speed signal acquisition, and an increase in the period of autonomous operation. Other important applications include surveillance, navigation, missile guidance, secure communications, identification friend-or-foe, and electronic warfare. ${ }^{2}$

The emerging applications require new compact time-distribution systems technologies capable of achieving signal phase (time) common synchronization of better than $10^{-9}$ sec relative to the Coordinated Universal Time (UTC) standard; intersystem synchronization of less than $10^{-8}$ sec relative to battle group; and less than $10^{-9}$ sec for interoperability, surveillance, and high-speed communications. ${ }^{1}$

Solid-state and atomic oscillators are the key components enabling time and frequency distribution for communication, navigation, and command and control systems.

The quality of time-distribution system is defined by the precision of time synchronization within a prescribed interval of time. To support the emerging applications we are interested in clocks with (i) signal phase (time) communication synchronization less (better) than $28 \mathrm{~ns}$ within 5 min (real time), UTC; (ii) intersystem synchronization less (better) than $28 \mathrm{~ns}$ relative to other system nodes within 5 min (real time); and (iii) local navigation/communication systems capable of time transfer less (better) than $28 \mathrm{~ns}$, UTC.

The operational frequency mismatch $(\Delta f / f)$, where $f$ is a nominal frequency and $\Delta f$ is a frequency deviation from the nominal, is a measure of oscillator quality and subsequently the quality of the frequency distribution system. Different applications can tolerate different levels of frequency mismatches. For example, for lowaccuracy aircraft/land mobile platforms, the requirement for frequency mismatch is $10^{-12}$, while for intermediate land reference sites the requirement is an order of magnitude higher, $10^{-13}$. For large Time Division Multiple Access (TDMA) systems, the tolerable frequency mismatch is on the order of $10^{-11}$.

Small Size, Weight, and Power (SWaP) are critical metrics for portable time and frequency distribution systems. The target performance characteristic for low-power clocks and oscillators is long-term stability (aging), which need to be less than $10^{-11} /$ month, with less than $1 \mathrm{~W}$ power consumption. It is desirable that the 
oscillators have small SWaP and preserve the level of long-term stability while surviving the inertial environment with accelerations on the level $10,000 g$, where $g$ is the gravity constant.

For comparison, the one-way satellite transmission from a GPS satellite in common view at two sites allows one to do accurate time transfer to within $10 \mathrm{~ns}$, with a potential to achieve accurate time transfer of the order of $1 \mathrm{~ns}$, Ref. 3. Achieving an accuracy of time transfer on the level of $1 \mathrm{~ns}$ is loosely defined as "precision timing."

\subsection{Inertial Navigation Systems}

An Inertial Navigation System (INS) is a self-contained, covert system that provides continuous estimates of some or all components of a vehicle state, such as position, velocity, acceleration, attitude, angular rate, and often guidance or steering inputs. The linear position and angular orientation are supplied to the INS by Inertial Measurement Units (IMUs). The navigation-grade performance provided by inertial sensors is defined as an INS that accumulates an uncertainty in location not greater than one nautical mile (nmi), or $1.852 \mathrm{~km}$, after one hour of navigation. The error in position is historically defined by the Circular Error Probable (CEP), an intuitive measure of a weapon system's accuracy defining a circle within which half of a missile's projectiles are expected to fall. That is, if the CEP is $\mathrm{n}$ meters, $50 \%$ of fired rounds land within $\mathrm{n}$ meters of the target. The ability to achieve a CEP of $1 \mathrm{nmi}$ in one hour (or $1 \mathrm{nmi} /$ hour) does not translate to a unique performance requirement for a gyroscope and/or an accelerometer. Rather, it presents a trade-off in the overall IMU error budget. The trades can be generated within a family of gyroscope errors, such as gyro Angle Random Walk (ARW) vs. bias drift, or similarly within a family of accelerometer errors. For example, an IMU with gyroscope bias drift of $0.01^{0} /$ hour combined with an accelerometer bias drift of $25 \mu \mathrm{g}$ would guarantee a CEP of $<1 \mathrm{nmi} /$ hour, if no other errors are present. To generate the trade-off space for component performance, one efficient approach is to first generate the parameter space at the linear error covariance level, taking into account the bias drift of components, and subsequently perform a more extensive modeling in a bounded trade-off space by a nonlinear Monte Carlo simulation.

The ability to navigate and keep precise timing has been an important factor in defining the military and economic power of nations for at least a millennium. For almost a century, the development of high-performance inertial instruments has been a very extensive area of research. ${ }^{4}$ It is anticipated that the following level of performance will soon be achieved, significantly reducing navigation errors and enhancing military capabilities, within the next 5 to 10 years: $^{1}$ (i) $<0.1 \mathrm{nmi} /$ hour CEP for aircraft, vehicle, or spacecraft for attitude, guidance, and control; (ii) $<1.0 \mathrm{nmi}$ in 30 hours for ships; and (iii) $<0.4 \mathrm{nmi} /$ hour CEP for missiles. It is critical that INS systems of future generations be capable of operating through shock levels greater than $1,000 \mathrm{~g}$.

Similarly to clocks, the reduction of $\mathrm{SWaP}$ and cost $(\mathrm{SWaP}+\mathrm{C})$, while not compromising in performance, are the critical metrics for future development of IMUs. The current performance of state-of-the-art MEMS-based IMUs is on the level of tactical grade, with CEP approaching $100 \mathrm{nmi} /$ hour. There is a great potential for achieving performance improvements that will subsequently enable platforms for personal navigation, precision navigation of small Unmanned Aerial Vehicles (UAVs), Unmanned Underwater Vehicles (UUVs), and GPS-free navigators for missiles. It is expected that the performance levels of chip-scale inertial instruments and clocks, shown in Table 1, could be achieved within the next 5 to 10 years, thus significantly enhancing military capabilities. The "conservative" estimations are projected by the Department of Defense's Science and Technology List for Positioning Navigation and Timing. ${ }^{1}$ The "aggressive" estimates are projected by successful completion of the micro-PNT program described in this paper.

The military has access to a currently specified accuracy of $21 \mathrm{~m}$ (95\% probability) from the GPS Precise Positioning Service (PPS). Accuracy can be improved after calibration for some of the GPS errors, e.g., by utilizing optimal estimation techniques correlating GPS and INS signals. A CEP of less than $10 \mathrm{~m}$ has been routinely achieved, with a potential to achieve accurate positioning of the order of $1 \mathrm{~m}$ CEP.

Navigation, guidance, and automatic control are not the only military applications that could benefit from improvements in inertial sensors. Azimuth or north-pointing determination systems include celestial devices, magnetic compasses, and inertial sensors. Utilization of gyroscopes to precisely determine orientation has a number of benefits attributed to their immunity to magnetic fields, speed of acquisition, and potentially small $\mathrm{SWAP}+\mathrm{C}$. For this purpose, a variety of inertial equipment is being explored, including Inertial Measurement 
Table 1. Projected performance of chip-scale inertial sensors and clocks by 2015.

\begin{tabular}{|c|c|c|c|}
\hline Key Parameters & Units & Conservative & Aggressive \\
\hline \multicolumn{4}{|l|}{ Gyroscopes } \\
\hline Angle Rate Bias Stability $(1 \sigma)$ & 0 /hour & 0.3 & 0.01 \\
\hline Drift rate stability & $0 /$ hour & 1 & 0.1 \\
\hline Scale factor $(1 \sigma)$ & $\mathrm{ppm}$ & 100 & 100 \\
\hline Range of Operation & $0 / \mathrm{sec}$ & $\pm 1,440$ & $\pm 15,000$ \\
\hline Power Consumption & $\mathrm{mW}$ & $<50$ & 5 \\
\hline \multicolumn{4}{|l|}{ Accelerometers } \\
\hline Acceleration Bias Stability $(1 \sigma)$ & $\mu g$ & 130 & 100 \\
\hline Scale Factor $(1 \sigma)$ & $\mathrm{ppm}$ & 130 & 100 \\
\hline \multicolumn{4}{|l|}{ Inertial measurement units } \\
\hline Navigation Error (CEP) & nmi/hour & 5 & 1 \\
\hline Power Consumption & $\mathrm{W}$ & $<0.25$ & $<0.2$ \\
\hline \multicolumn{4}{|l|}{ Chip-scale clocks } \\
\hline Time loss at $1 \mathrm{~min}$ & $\mathrm{~ns}$ & 10 & 1 \\
\hline Time loss at 1 hour & ns & 50 & 1.2 \\
\hline Time loss at 1 day & $\mathrm{ns}$ & 100 & 5 \\
\hline Time loss at 1 month & ns & 1000 & 30 \\
\hline Frequency retrace & - & $10^{-11}$ & $10^{-13}$ \\
\hline \multicolumn{4}{|l|}{ Environmental characteristics } \\
\hline Performance through shock & $\mathrm{g}$ & 1,000 & 1,000 \\
\hline Survive shock & $\mathrm{g}$ & 10,000 & 20,000 \\
\hline
\end{tabular}

Units (IMU), Attitude Heading Reference Systems (AHRS), and gyro-compasses. Providing an azimuth or north-pointing accuracy of less (better) than 0.5 arc minute multiplied by secant latitude has the potential to significantly enhance military capabilities for many targeting applications, especially for anticipated mobile platforms.

\section{CURRENT RESEARCH}

This section provides an overview of the ongoing efforts funded by DARPA/MTO under the micro-PNT program.

\subsection{Clocks}

The potential payoff of the precision clock technology developed by the program will enable ultra-miniaturized and ultra-low power absolute time and frequency references for applications such as nano/pico satellite systems, UUVs, UAVs, wristwatch-size high-security UHF communicators, and jam-resistant GPS receivers.

There are currently two efforts within the micro-PNT program involving the development of clocks: the Chip-Scale Atomic Clock (CSAC) and the Integrated Micro Primary Atomic Clock Technology (IMPACT).

The goal of the CSAC effort is to create ultra-miniaturized, low-power, atomic time and frequency reference units that will achieve, relative to present approaches: more than $200 \times$ reduction in size (from $230 \mathrm{~cm}^{3}$ to ¡1 $\mathrm{cm}^{3}$ ); more than $300 \times$ reduction in power consumption (from $10 \mathrm{~W}$ to less than $30 \mathrm{~mW}$ ); and matching performance $\left(1 \times 10^{-11}\right.$ accuracy and $1 \mu \mathrm{sec} /$ day stability). This work, funded by DARPA since 2002 , has been supporting 11 teams. The program is currently in its final phase and supports two performers - Symmetricom 
and Rockwell. Symmetricom has already demonstrated pilot units that are $1 \mathrm{~cm}^{3}$ in volume, consume on the order of $100 \mathrm{~mW}$ of power, and perform on the level of better than $30 \times 10^{-11}$ short-term 1 sec instability (Allan Deviation) and $5 \times 10^{-11} /$ day $\left(1.4 \times 10^{-10} / \mathrm{month}\right)$ long-term frequency drift. Results and intellectual property produced by CSAC can be found in Refs. 5-33. The IMPACT program seeks to improve the stability and accuracy of microscale atomic clocks. The performance of atomic clocks is affected by buffer gases $\left(N_{2}\right.$ or $A r$ ), which are necessarily present in either Rb- or Cs-based atomic clocks. Buffer gas atoms interact with alkali atoms and effectively shift the resonant frequency of atoms. Emerging atomic clock technologies based on laser-cooled atoms and trapped ions could eliminate the limitations of CSAC.

The goal of IMPACT is to create miniaturized, low-power, integrated micro primary atomic clock technology that will achieve significant reduction in size relative to conventional clocks, but slightly larger than CSAC (volume less than $5 \mathrm{~cm}^{3}$ in final package, excluding battery); significant reduction in power relative to conventional clocks, but slightly greater than CSAC $(50 \mathrm{~mW})$; and an order of magnitude increase in performance relative to CSAC (frequency accuracy $1 \times 10^{-13}$, Allan deviation at one-hour integration time, and stability characterized by $5 \mathrm{nsec}$ /day time loss). The work has been funded by DARPA since 2008, and currently supports four teams - Honeywell, Symmetricom, Sandia National Lab, and OE Waves. The overall approach is based on sampling of atomic transitions at extremely low temperatures, requiring vacuum on the level of $10^{-9}$ Torr and the ability to trap atoms in a small volume. The technology has been previously demonstrated on a large scale, but transferring the technology to small scale is far from trivial, requiring major innovations. The effort has already demonstrated Magneto-Optical Trapping in a $16 \mathrm{~cm}^{3}$ atomic cell and chip-scale clocks implemented using cold atoms performing on the level, $Q \times S / N \sim 2.6 \times 10^{10}$, time loss after $1 \mathrm{~ms}$ equal to $10^{-4} \mathrm{~ns}$, after $1 \mathrm{~s}-6 \times 10^{-3} \mathrm{~ns}$, after 1 hour - less than $10 \mathrm{~ns}$, and after 24 hours - on the order of $100 \mathrm{~ns}$. Frequency retrace was demonstrated at the end of the phase on the level of $10^{-11}$.

\subsection{Inertial Sensors and Systems}

There are currently three efforts within the PNT program involving the development of inertial sensors and systems - the Navigation-Grade Integrated Micro Gyroscopes (NGiMG), the Micro Inertial Navigation Technology (MINT), and the Information Tethered Micro Automated Rotary Stages (IT-MARS).

The NGiMG program seeks to develop tiny, low-power, rotation-rate sensors capable of achieving performance commensurate with requirements for GPS-denied navigation of small platforms, including individual soldiers, unmanned (micro) air vehicles, unmanned underwater vehicles, and even tiny (e.g., insect-sized) robots. By

harnessing the advantages of microscale miniaturization, the NGiMG program is expected to yield tiny (if not chip-scale) gyroscopes with navigation-grade performance characteristics: overall size less than $1 \mathrm{~cm}^{3}$ (no power source), power consumption less than $5 \mathrm{~mW}$, angle random walk (ARW) less than $0.001^{0} / \sqrt{\text { hour }}$, bias drift less than $0.01^{0} /$ hour , scale factor stability less than $50 \mathrm{ppm}$, full-scale range greater than $500^{\circ} / \mathrm{sec}$, and bandwidth on the order of $300 \mathrm{~Hz}$.

The NGIMG effort has been funded by DARPA since 2005, and work is currently being conducted by three teams - Northrop Grumman, Boeing, and Archangel. The effort has demonstrated several experimental prototypes (some, but not all, independently verified by the government) performing on the level of ARW $0.01^{0} / \sqrt{\text { hour }}$ and bias drift $0.05^{0} /$ hour .

The MINT effort seeks to develop microscale low-power navigation sensors that allow long-term, hours to days, precisions navigation in GPS-denied environment. The goal is to create high-precision, navigation-aiding sensors that directly measure intermediate inertial variables, such as velocity and distance, to mitigate the error growth encountered by integrating signals from accelerometers and gyroscopes alone. In addition to aiding sensors such as velocity sensors, the combination of microscale inertial sensors are to be integrated to a formfactor of one or two integrated circuits. Such an integrated sensor suite will be incorporated into the sole of a shoe for accurate and precise velocity sensing using zero velocity events during walking. The final goal of MINT is to achieve an overall packaging and form-factor for velocity sensor (excluding IMU) of less than $1 \mathrm{~cm}^{3}$, power consumption for the velocity sensor of less than $5 \mathrm{~mW}, 1-\mathrm{m}$ position accuracy after 36 hours of walking, and 10 $\mu \mathrm{m} / \mathrm{s}$ velocity sensing bias per step. The effort has been funded by DARPA since 2008 and currently involves work by four teams - Carnegie Mellon, Analog Devices, Northrop Grumman, and Case Western/University of Utah. To date, the effort has demonstrated position error on the order of $3 \mathrm{~m}$ after $30 \mathrm{~min}$ of walking. 


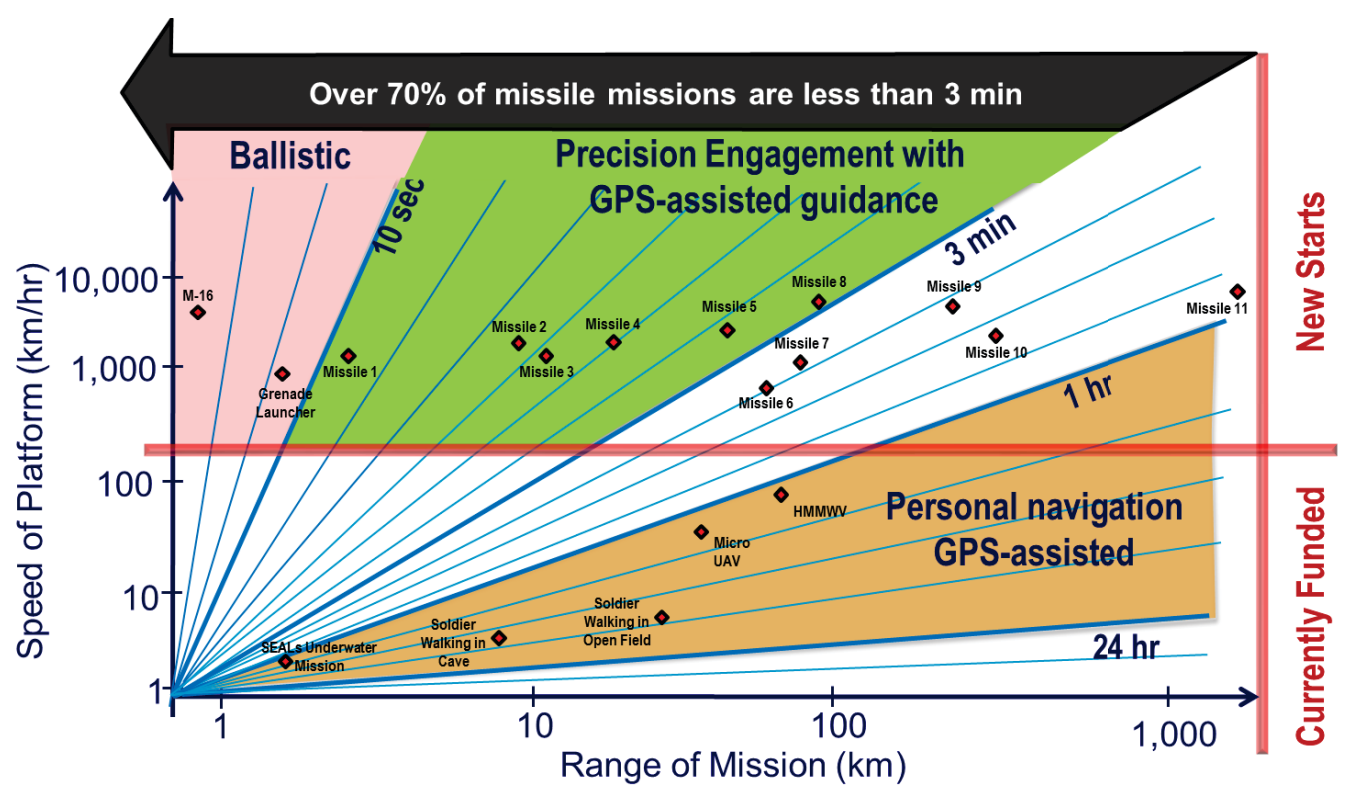

Figure 1. Range of missions vs. speed of platforms for common DoD needs. The duration of missions is ranging from seconds to minutes to hours. Majority of missile missions are fast, but less than 180 seconds ( 3 minutes) in duration. All missions with duration greater than $10 \mathrm{sec}$ are typically utilizing GPS. micro-PNT program develops a single chip solution enabling self-contained inertial navigation (without the need for external signals such as GPS) for diverse DoD scenarios.

The goal of the IT-MARS program is to implement and demonstrate a MEMS-fabricated rotary stage providing a rotational degree of freedom to planar MEMS structures and sensors, thus enabling free rotation of micro-structures and micro-sensors relative to the package, with coupled power and signal transfer from the rotating platform to the package. The IT-MARS effort may enable highly accurate calibration of inertial sensors and serve as a micro-platform for carouseling of inertial sensors that further enable on-chip calibration and gyro compassing. The final goal of the program is to achieve an overall volume of no more than $1 \mathrm{~cm}^{3}$, power consumption for actuation on the order of $10 \mathrm{~mW}$, angle position absolute accuracy to within 1 milli-degree, maximum wobble of 10 micro-radians, a rotation rate of $360 \mathrm{deg} / \mathrm{sec}$, and reliability (run time of rotor) better than $10^{4}$ hours. This effort, which has been funded by DARPA since 2009, currently supports three teams - UCLA, UC Berkeley, and the Boyce Thompson Institute. The work has already demonstrated free rotated platforms, and future efforts will be focused on manufacturability and precision control of the stage - rotation and reduction of wobbling.

\section{NEW INITIATIVES}

In January 2010, DARPA/MTO launched a coordinated effort focused on the development of microtechnology specifically addressing the challenges associated with miniaturization of high-precision clocks and inertial instruments. The new program, Microtechnology for Positioning, Navigation, and Timing (micro-PNT), aggregated the existing efforts (CSAC, IMPACT, NGIMG, MINT, and IT-MARS) and initiated four complementary new developments: (1) Microscale Rate Integrating Gyroscopes(MRIG), (2) Chip-Scale Timing and Inertial Measurement Unit (TIMU), (3) Primary and Secondary Calibration on Active Layer (PASCAL), and (4) Platform for Acquisition, Logging, and Analysis of Devices for Inertial Navigation \& Timing (PALADIN\&T).

The overall goal of the new aggregated micro-PNT program is to focus all of these complementary efforts toward achieving one specific overarching goal - self-contained chip-scale inertial navigation, Figure 1. The reduction of Size, Weight, and Power and Cost $(\mathrm{SWaP}+\mathrm{C})$ of Inertial Measurement Units (IMUs) and Timing Units (TUs) is the technological objective. The developments consider a number of operational scenarios, ranging from dismounted solder navigation to navigation, guidance, and control (NGC) of UAVs/UUVs and guided 
missiles. The new micro-PNT initiatives will increase the dynamic range of inertial sensors, addressed by the new MRIG effort; reduce the long-term drift in clocks and inertial sensors, addressed by the PASCAL work; develop ultra-small chips providing position, orientation, and time information, addressed by the TIMU effort; and provide a universal and flexible platform for the testing and evaluation of components developed within the comprehensive micro-PNT program, addressed by the PALADIN\&T effort.

The primary goal of MRIG is to create a vibratory gyroscope that can be instrumented to measure the angle of rotation directly, thereby extending the dynamic range and eliminating the need for integrating the angular rate information; MRIG will thus eliminate the accumulation of errors due to numerical/electronic integration. The final goals are (1) to extend the dynamic range to $15,000 \mathrm{deg} / \mathrm{sec}$; (2) achieve drift repeatability on the level of $0.1 \mathrm{deg} / \mathrm{hour}$ (angle dependent) and $0.01 \mathrm{deg} / \mathrm{hour}$ (bias-dependent) under variable $-55^{0} \mathrm{C}$ to $85^{\circ} \mathrm{C}$ thermal variations; (3) and achieve the Angle Random Walk of $0.001 \mathrm{deg} / \sqrt{\mathrm{hour}}$, an operation range of 1,000 $\mathrm{g}$ with acceleration sensitivity of $10^{-5} \mathrm{deg} / \mathrm{hour} / \mathrm{g}$, vibration sensitivity angle random walk of $0.01 \mathrm{deg} / \sqrt{\mathrm{hour}}$ per $g / \sqrt{H z}$, and drift rate of $0.01 \mathrm{deg} /$ hour per $g^{2} / \sqrt{\mathrm{Hz}}$. These performance characteristics are thought to be achievable through development of precision 3-D fabrication technologies utilizing high-Q materials; development of wafer-level balancing and trimming techniques that reduce the effects of aniso-inertia (mass misbalance), anisocompliance (stiffness misbalance), and aniso-damping (damping misbalance); and development of active control and an active calibration architecture. The following performers have be selected for the initial phase of the program: Draper Labs, Honeywell, Northrop Grumman, Systron Donner, UC Irvine, UC Davis, UCLA, Cornell, University of Michigan, Yale University.

PASCAL will develop self-calibration technologies intended to eliminate the long-term bias drift of inertial sensor and clocks. The grand challenge of this effort is to raise long-term bias stability to the level of $1 \mathrm{ppm}$. This level of stability represents a two orders of magnitude improvement compared to state-of-the-art inertial micro-sensors, which are currently at $200 \mathrm{ppm} .^{34}$ The work will investigate an approach for fabricating sensors on an active layer that may serve as a calibration layer for micro-PNT systems.

The TIMU program will address challenges associated with the development of a miniature $\left(10 \mathrm{~mm}^{3}\right)$, lowpower $(200 \mathrm{~mW})$, high-performance (CEP on the order of $1 \mathrm{nmi} /$ hour $)$, and self-sufficient navigation system on-a-chip. The smallest state-of-the-art IMUs perform on the level of tactical-grade instruments (CEP on the order of $100 \mathrm{nmi} /$ hour) and are about the size of an apple (more than $10^{4} \mathrm{~mm}^{3}$ ). This effort intends to develop a technological foundation for a navigation-grade TIMU (CEP less than $1 \mathrm{nmi} / \mathrm{hour}$ and time accuracy of 1 $n s / \min )$ with a significant reduction in SWaP, potentially miniaturizing the entire TIMU to the size of an apple seed $\left(10 \mathrm{~mm}^{3}\right)$.

PALADIN\&T effort will develop a universal platform for test and evaluation of prototypes developed within the micro-PNT program. The effort will also simplify the uniform evaluation of pilot prototypes within the micro-PNT program and provide an early field demonstration of the technology, thus advancing the Technology Readiness (TR) level.

\section{CONCLUSIONS}

The duration of military missions ranges from minutes to hours to days, and most missions require extreme PNT accuracy throughout their entire duration - accuracy in position to within $10 \mathrm{~m}$, and accuracy in time on the order of $1 \mathrm{~ns}$. Current state-of-the-art microscale clocks and inertial instruments can provide the required level of precision only for missions having a duration of no more than about one minute. The micro-PNT program at DARPA is developing small $\mathrm{SWaP}+\mathrm{C}$ inertial sensors for a variety of operational scenarios for missions ranging from minutes to hours, Figure 1. The current projects (CSAC, IMPACT, NGIMG, MINT, ITMARS) are mainly focused on navigation, characterized as missions of prolonged durations in relatively benign environments (a few hours of operation on a platform moving at relatively low speed, less than $100 \mathrm{~km} / \mathrm{hour}$ ). The new initiatives (MRIG, TIMU, PASCAL, and PALADIN) are targeting the challenges of missile guidance for precision engagement scenarios, short duration missions in highly dynamic environments (10 sec to $3 \mathrm{~min}$ of operation at speeds of $1,000 \mathrm{~km} /$ hour and higher). The ongoing efforts and new initiatives explore new physical phenomena, high-quality factor materials, highly specialized fabrication technologies, and innovative system integration approaches. 


\section{DISCLAIMER}

The views, opinions, and/or findings contained in this article/presentation are those of the author/presenter and should not be interpreted as representing the official views or policies, either expressed or implied, of the Defense Advanced Research Projects Agency or the Department of Defense. This document was cleared by DARPA on October 28, 2010. All copies should carry the Distribution Statement "A" (Approved for Public Release, Distribution Unlimited).

\section{REFERENCES}

[1] "Developing science and technologies list, section 16: Positioning, navigation and timing technology," 2009. Department of Defense, http://www.dtic.mil/mctl/MCTL/Sec16MCTLg.pdf.

[2] J. Vig, "Military applications of high accuracy frequency standards and clocks," IEEE Trans. on Ultrasonics, Ferroelectrics, and Frequency Control 40, pp. 522-527, 1993.

[3] D. Allan and M. Weiss, "Accurate time and frequency transfer during common-view of a gps satellite," Proc. 34th Ann. Freq. Control Symposium, pp. 334-346, 1980. USAERADCOM. Ft. Monmouth, NJ.

[4] D. MacKenzie, Inventing Accuracy, The MIT Press, 2000.

[5] J. Kitching, S. Knappe, and L. Hollberg, "Miniature vapor-cell atomic-frequency references," Applied Physics Letters 81, pp. 553-5, 15 July 2002.

[6] S. Knappe, V. Velichansky, H. Robinson, L. Liew, J. Moreland, J. Kitching, and L. Hollberg, "Atomic vapor cells for miniature frequency references," in Proceedings of the 2003 IEEE International Frequency Control Symposium and PDA Exhibition. Jointly with the 17th European Frequency and Time Forum (IEE Cat. No.03CH37409), pp. 31-2, 20032003.

[7] S. Knappe, P. Schwindt, V. Shah, L. Hollberg, J. Kitching, L. Liew, and J. Moreland, "Microfabricated atomic frequency references," in Proceedings of the 2004 IEEE International Frequency Control Symposium And Exhibition (IEEE Cat. No.04CH37553C), M. Yuhas, ed., pp. 87-91, 20042004.

[8] L.-A. Liew, S. Knappe, J. Moreland, H. Robinson, L. Hollberg, and J. Kitching, "Micromachined alkali atom vapor cells for chip-scale atomic clocks," in 17th IEEE International Conference on Micro Electro Mechanical Systems. Maastricht MEMS 2004 Technical Digest (IEEE Cat. No.04CH37517), pp. 113-16, 20042004.

[9] L.-A. Liew, S. Knappe, J. Moreland, H. Robinson, L. Hollberg, and J. Kitching, "Microfabricated alkali atom vapor cells," Applied Physics Letters 84, pp. 2694-6, 5 April 2004.

[10] M. Zhu, L. Cutler, J. Berberian, J. DeNatale, P. Stupar, and C. Tsai, "Narrow linewidth CPT signal in small vapor cells for chip scale atomic clocks," in Proceedings of the 2004 IEEE International Frequency Control Symposium And Exhibition (IEEE Cat. No.04CH37553C), M. Yuhas, ed., pp. 100-3, 20042004.

[11] J. Kitching, S. Knappe, L. Liew, P. Schwindt, K. Shah, J. Moreland, and L. Hollberg, "Microfabricated atomic clocks," in 18th IEEE International Conference on Micro Electro Mechanical Systems (IEEE Cat. No.05CH37610), pp. 1-7, 20052005.

[12] C.-C. Nguyen and J. Kitching, "Towards chip-scale atomic clocks," in Proceedings of IEEE International Solid-State Circuits Conference, 2005. San Francisco, CA.

[13] S. Knappe, V. Gerginov, P. Schwindt, V. Shah, H. Robinson, L. Hollberg, and J. Kitching, "Atomic vapor cells for chip-scale atomic clocks with improved long-term frequency stability," Optics Letters 30, pp. 2351-3, 15 September 2005.

[14] M. Mescher, R. Lutwak, and M. Varghese, "An ultra-low-power physics package for a chip-scale atomic clock," in TRANSDUCERS '05. The 13th International Conference on Solid-State Sensors, Actuators and Microsystems. Digest of Technical Papers (IEEE Cat. No. 05TH8791), pp. 311-16 Vol. 1, 20052005.

[15] R. Lutwak, P. Vlitas, M. Varghese, M. Mescher, D. Serkland, and G. Peake, "The MAC - a miniature atomic clock," in 2005 IEEE International Frequency Control Symposium and Exhibition, p. 6 pp., 20052005.

[16] S. Romisch and R. Lutwak, "Low-power, 4.6-GHz, stable oscillator for CSAC," in Proceedings of the 2006 IEEE International Frequency Control Symposium and Exposition, p. 4 pp., 20062006.

[17] D. Serkland, G. Peake, K. Geib, R. Lutwak, R. Garvey, M. Varghese, and M. Mescher, "VCSELs for atomic clocks," in Proceedings of the SPIE - The International Society for Optical Engineering, 6132, pp. 6132081-11, 9 February 2006. 
[18] S. Knappe, P. Schwindt, V. Gerginov, V. Shah, A. Brannon, B. Lindseth, L.-A. Liew, H. Robinson, J. Moreland, Z. Popovic, L. Hollberg, and J. Kitching, "Chip-scale atomic devices at NIST," in Proceedings of the SPIE - The International Society for Optical Engineering, 6604, pp. 660403-1-8, 20072007.

[19] S. Knappe, V. Shah, A. Brannon, V. Gerginov, H. Robinson, Z. Popovic, L. Hollberg, and J. Kitching, "Advances in chip-scale atomic frequency references at NIST," Proceedings of the SPIE - The International Society for Optical Engineering 6673, pp. 1-10, 13 September 2007.

[20] R. Lutwak, A. Rashed, D. Serkland, G. Peake, M. Varghese, G. Tepolt, J. Leblanc, and M. Mescher, "The miniature atomic clock - pre-production results," in 2007 IEEE International Frequency Control Symposium Jointly with the 21st European Frequency and Time Forum, pp. 1327-33, 20072007.

[21] D. Serkland, K. Geib, G. Peake, R. Lutwak, A. Rashed, M. Varghese, G. Tepolt, and M. Prouty, "VCSELs for atomic sensors," in Proceedings of the SPIE - The International Society for Optical Engineering, 6484, pp. 648406-1-10, 8 February 2007.

[22] D. Youngner, L. Lust, D. Carlson, S. Lu, L. Forner, H. Chanhvongsak, and T. Stark, "A manufacturable chip-scale atomic clock," in TRANSDUCERS '07 8 Eurosensors XXI. 200714 th International Conference on Solid-State Sensors, Actuators and Microsystems, pp. 82-7, 20072007.

[23] A. M. Braun, T. J. Davis, M. H. Kwakernaak, J. J. Michalchuk, A. Ulmer, W. K. Chan, J. H. Abeles, Z. A. Shellenbarger, Y.-Y. Jau, and W. Happer, "RF-Interogated End-State Chip-Scale Atomic Clock," in 39th Annual Precise Time and Time Interval (PTTI) Meeting, November 2007. Long Beach, CA.

[24] J. DeNatale, R. Borwick, C. Tsai, P. Stupar, Y. Lin, R. Newgard, R. Berquist, and M. Zhu, "Compact, low-power chip-scale atomic clock," in 2008 IEEE/ION Position, Location and Navigation Symposium PLANS 2008, pp. 67-70, 5 May 2008.

[25] R.Lutwak, "Chip scale atomic clocks tutorial," the IEEE International Frequency Control Symposium (FCS'08), 2008. Honolulu, HI.

[26] R. Lutwak, "The chip-scale atomic clock - recent developments," in 2009 Joint Meeting of the European Frequency and Time Forum (EFTF'09) and the IEEE International Frequency Control Symposium (FCS'09), pp. 573-7, 2009.

[27] R.Lutwak, "Chip scale atomic clocks," Workshop on Integrated Atomic Systems II (IAS II), in conjunction with the 11th Annual Southwest Quantum Information and Technology (SQuInT) Workshop, 2009. Seattle, Washington.

[28] A. Laws, I. Borwick, R., P. Stupar, J. DeNatale, and Y. Lee, "Thermal and structural analysis of a suspended physics package for a chip-scale atomic clock," Journal of Electronic Packaging 131, p. 041005 (9 pp.), December 2009.

[29] G. Tepolt, M. Mescher, J. LeBlanc, R. Lutwak, and M. Varghese, "Hermetic vacuum sealing of MEMS devices containing organic components," in Proceedings of the SPIE - The International Society for Optical Engineering, 7592, p. 759207 (12 pp.), 20102010.

[30] NIST Patents: US 6806784, US 6831522.

[31] Symmetricom Patents: US 7064835, US 6605849, US 7215213, US 6318621, US 6261866.

[32] Honeywell Patents: US 7494598, US 7379486.

[33] Teledyne Patent: US 7619485.

[34] M. Weinberg and A. Kaurepenis, "Error sources in in-plane silicon tuning-fork mems gyroscopes," IEEE J. of Microelectromechanical Systems 15(3), pp. 479-491, 2006. 